This item was submitted to Loughborough's Research Repository by the author.

Items in Figshare are protected by copyright, with all rights reserved, unless otherwise indicated.

\title{
Individual-level change in achievement goals in physical education
}

PLEASE CITE THE PUBLISHED VERSION

http://dx.doi.org/10.5861/ijrse.2016.1418

\section{PUBLISHER}

Consortia Academia (@ the authors)

\section{VERSION}

VoR (Version of Record)

\section{PUBLISHER STATEMENT}

This work is made available according to the conditions of the Creative Commons Attribution-NonCommercialNoDerivatives 4.0 International (CC BY-NC-ND 4.0) licence. Full details of this licence are available at: https://creativecommons.org/licenses/by-nc-nd/4.0/

\section{LICENCE}

CC BY-NC-ND 4.0

\section{REPOSITORY RECORD}

Warburton, Victoria E., and Christopher M. Spray. 2019. "Individual-level Change in Achievement Goals in Physical Education”. figshare. https://hdl.handle.net/2134/20724. 
Individual-level change in achievement goals in physical education

Warburton, Victoria E. $₫$

University of East Anglia, United Kingdom (v.warburton@uea.ac.uk)

Spray, Christopher M.

Loughborough University, United Kingdom (c.m.spray@lboro.ac.uk)

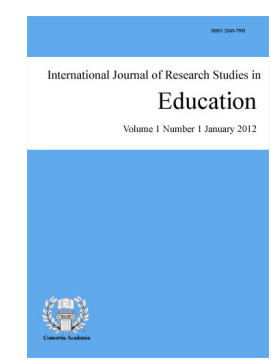

ISSN: 2243-7703 Online ISSN: 2243-7711

Received: 15 January 2016

\section{Abstract}

We examined different methods of assessing individual-level stability and change in goal adoption within the context of school physical education (PE) over the transition from primary to secondary school and between school years in Key Stage 3. We assessed whether implicit theories of ability and gender were associated with individual-level change and ipsative continuity. Data from two longitudinal samples in which pupils completed self-reports of achievement goal adoption and implicit theories of ability are presented. Across both samples there was evidence of both goal stability and change across the key transition periods and some evidence for the role of implicit theories of ability in goal stability and change at the individual-level. Pupil gender was not associated with stability and change in goals in a consistent manner. Implications for future research are discussed.

Keywords: individual change; physical education; achievement goals; transitions 


\section{Individual-level change in achievement goals in physical education}

\section{Introduction}

The achievement goal literature is rich with research across a range of different achievement contexts that identifies the antecedents and consequences of adopting particular achievement goals (see Elliot, 1999, 2005; Harwood, Spray \& Keegan, 2008; Roberts, 2012). However, in recent years, the focus of research interest has been on understanding changes in achievement goal adoption and the factors associated with such changes (e.g., Fryer \& Elliot, 2007; Muis \& Edwards, 2009; Senko \& Harackiewicz, 2005; Spray, Warburton, \& Stebbings, 2013; Warburton \& Spray, 2008, 2009). In the educational domain this research has utilised a variety of analytic techniques to assess goal stability and change across specific tasks (e.g., Fryer \& Elliot, 2007; Muis \& Edwards, 2009; Senko \& Harackiewicz, 2005), across the transition from primary school (e.g., Anderman \& Anderman, 1999; Urdan \& Midgley, 2003), across a school year (Bong, 2005), and throughout college (Corker, Donnellan \& Bowles, 2013). Although research in the physical domain has examined change in achievement goal adoption across the transition from primary school and within Key Stage 3 at secondary school (e.g., Spray et al., 2013; Warburton \& Spray, 2008, 2009) it has focused mainly on mean-level change. Key questions concerning other indicators of change, particularly at the individual-level therefore remain underexplored in the context of school physical education (PE). In this paper, we examine different methods of assessing individual-level change in goal adoption within PE. Young people in PE are often engaged in achievement activities over a period of time, and there is the potential for change in their motivation to occur. Thus, PE would appear to be a particularly fruitful setting for exploring individual-level change.

Work by Elliot and colleagues (see Elliot, 1999, 2005, for reviews), in delineating a more differentiated achievement goal construct, provides the opportunity to explore interesting questions regarding changes in achievement goal adoption. Elliot and colleagues' work suggests that achievement goals represent the aim of achievement behaviour, and in the 2 × 2 achievement goal framework (Elliot \& McGregor, 2001), they highlight the possibility of endorsing four achievement goals that differ based on the definition and valence of competence. In this framework, mastery-approach goals (MAp) focus on developing task- or self-referent competence, whereas mastery-avoidance goals (MAv) focus on avoiding developing task- or self-referent incompetence. Thus, individuals may strive to do their best on a task, or alternatively, their striving may stem from a concern that they are unable to do the task as well as they feel they can. Performance-approach goals (PAp) focus on demonstrating normative competence, whereas performance-avoidance goals (PAv) focus on avoiding demonstrating normative incompetence. Accordingly, individuals aim to show they are one of the best at a task, or alternatively, they are more concerned with being worse at the task than those around them. Individuals could endorse these goals in isolation or combination during an achievement situation and the processes of goal switching, changing the commitment to a different goal, or goal intensification, changing commitment to a single goal (Senko \& Harackiewicz, 2005), suggest the potential for complex temporal patterns of motivation during an achievement situation.

\subsection{Malleability in Achievement Goal Adoption}

The importance of taking a developmental perspective to understanding young people's achievement motivation has been underscored within both the educational (e,g., Fryer \& Elliot, 2007; Muis \& Edwards, 2009; Senko \& Harackiewicz, 2005) and sport psychology literatures (e.g., Barkoukis, Ntoumanis, Thøgersen-Ntoumani, 2010; Papaioannou, Bebetsos, Theodorakis, Christodoulidis, Kouli, 2006; Spray \& Warburton, 2011; Spray et al., 2013; Warburton \& Spray, 2008, 2009). Across the different contexts, this research has examined the temporal patterns of achievement motivation and while there appears to be some consistency with regards to reporting a decline in adaptive motivation (MAp goals), there are inconsistencies with regards to maladaptive motivation with some research reporting increases, and others decreases or stability. 
Further to this, the research has attempted to identify some of the antecedents and consequences which may be associated with stability and change in achievement goal adoption, such as fear of failure and gender (Fryer \& Elliot, 2007), implicit theories of ability (Warburton \& Spray, 2008, 2009), perceived competence (Papaioannou et al, 2006; Warburton \& Spray, 2008, 2009), motivational climate (Barkoukis et al., 2010), self-perceptions and self-esteem (Spray et al., 2013), intrinsic motivation (Papaioannou et al., 2006), and boredom and enjoyment (Barkoukis et al., 2010).

However, while research in the educational domain has utilised different analytic techniques to explore goal stability and change, the analysis of goal stability and change in the physical domain, with the notable exception of Conroy, Elliot and Hofer (2003), has tended to focus on mean-level change. This method of examining change over time leads to inferences that reflect a mean-level change for the whole sample. It is therefore not possible to discern the changes that occur at the individual level. Indeed, mean-level changes may mask individual-level changes to produce null results and conceal that a substantial proportion of individuals evidence change (Roberts, Caspi \& Moffitt, 2001). For example, increases over time in PAp goal adoption for one individual may cancel out decreases in PAp goal adoption for another individual and lead to the conclusion of no significant change over time in this goal. This finding will then be interpreted along with the other mean-level changes in achievement goal adoption to provide an overview and a basis for future intervention. Fryer and Elliot (2007) suggest exhibiting caution in making these inferences. When using different indicators of stability and change, i.e., differential continuity, mean-level change, individual-level change and ipsative continuity, the conclusions drawn from mean-level analyses can be different to those based on individual-level analyses. Consequently, mean-level analyses may not provide a complete understanding of changes in achievement goal adoption and the dynamics of motivational processes in achievement contexts.

\subsection{Individual-Level Change in Achievement Goal Adoption}

Conroy and colleagues (2003) used differential stability coefficients to examine relative changes in participant's true scores over a period of 21 days in a sample of recreational athletes. They found greater stability in within-person scores for PAp and PAv goals than both MAp and MAv goals. PAp goals exhibited the greatest stability, while the stability of MAp and MAv goals declined after 14 days and PAv goal stability declined after 21 days. The results are suggestive of stability in achievement goal adoption at the individual level. However, the reduced stability in achievement goal adoption after the 14 and 21 day period suggests that if individuals are engaged in achievement situations over longer periods of time one might expect more change than stability in achievement goal adoption.

Fryer and Elliot (2007) utilised a different indicator of individual change, the reliable change index (Christensen \& Mendoza, 1986; Jacobson \& Truax, 1991) and examined changes over a longer time period. Across the three studies reported, they found a greater proportion of university undergraduates were likely to report a decrease in MAp goals and an increase in PAv goals, whereas similar proportions were equally like to report an increase or decrease in PAp and MAv goals. Furthermore, Fryer and Elliot examined gender and fear of failure as predictors of individual-level change but found no consistent gender effects or any effect of fear of failure on individual change in achievement goal adoption. Muis and Edwards (2009) assessed the degree of similarity in the achievement task and its effect on individual-level changes in achievement goal adoption. Their results were supportive of Fryer and Elliot's, with greater stability for PAp goals and greater change being evident for MAp and PAv goals. Consequently, the findings from these studies suggest that PAp goals at the individual level appear to evidence stability rather than change and that there may be declines evident for MAp goal adoption over the course of an achievement task.

Fryer and Elliot (2007) also utilised ipsative continuity analyses which examine the configuration of goals within an individual. As the 2 x 2 framework highlights four potential achievement goals, ipsative continuity provides the opportunity to consider the multiple goals perspective (Barron \& Harackiewicz, 2001) and whether goal switching or goal intensification may be occurring. Fryer and Elliot found evidence of stability, suggesting 
that for undergraduate students, there was little change in the configuration of achievement goals within an individual over time. However, individuals with higher levels of fear of failure were more likely to experience a change in their configuration of goals. It was concluded that the influence of fear of failure on change in achievement goal adoption may be in the relative importance of achievement goals rather than in promoting change in individual goals (Fryer \& Elliot, 2007).

Drawing on the work of Dweck and colleagues (see Dweck, 1999 for an overview), Fryer \& Elliot (2007) propose that implicit theories of ability may be an interesting avenue for examining stability and change in achievement goal adoption due to the response of individuals to competence feedback. They suggested that endorsing an incremental belief, the belief that ability is a malleable, controllable quality that can be improved through learning, reduces these individuals' response to competence feedback and is likely to promote stability in goal adoption. On the other hand, an entity belief, the belief that ability is a fixed, stable capacity, is likely to promote malleability in goal adoption due to heightening young people's awareness of, and response to competence feedback.

Future research, across achievement contexts, which uses indicators of stability and change that incorporate individual-level analyses, will provide a complementary and comprehensive account of changes in achievement goal adoption. This will enable comparisons with findings from mean-level analyses to be developed. Furthermore, identifying predictors of individual-level changes in goal adoption will help to determine whether the factors prevalent at the sample level also emerge as important in predicting changes at the individual-level. Given that interventions aim to influence an individual's achievement goal adoption, this research may help to develop interventions which are more effective in bringing about positive changes in goal striving in achievement contexts.

\subsection{The Present Study}

The purpose of the present study was to extend the current literature on changes in motivation in the PE context by examining individual-level change and ipsative continuity over the transition from primary to secondary school and between school years in Key Stage 3. We also sought to examine whether two key antecedents of achievement goal adoption, namely incremental and entity beliefs, and also gender were associated with individual-level or ipsative change in achievement goal adoption. We constructed hypotheses based on the previous research on mean-level analyses in the PE context focusing only on that research which used the approach-avoidance framework (e.g., Spray et al., 2013; Warburton \& Spray, 2008, 2009). Based on this research, we anticipated that a greater proportion of pupils would report a decline than an increase in MAp goal adoption at each time point comparison. We did not offer, a priori, specific hypotheses associated with MAv, PAp and PAv goals due to the lack of consistency in mean-level findings for these goals in the PE context.

We also used the similarity in findings from the individual-level analyses from sport (Conroy et al., 2003) and education (Fryer \& Elliot, 2007; Muis \& Edwards, 2009) to construct hypotheses. We anticipated that pupils would evidence greater stability in PAp and MAv goals, while a greater proportion of pupils would report a decrease in MAp goal adoption or an increase in PAv goal adoption. For ipsative continuity, we expected the configuration of goals within individuals to evidence stability rather than change. We offered no specific a priori hypotheses for gender due to the lack of consistent findings in previous research; this was an exploratory aspect of the present study. Finally, drawing on the proposal of Fryer and Elliot (2007), we anticipated that pupils high in entity beliefs would be more likely to report change in their achievement goal adoption and configuration of goals, whereas those high in incremental beliefs would be more likely to report stability in their achievement goal adoption and configuration of goals. However, despite these a priori hypotheses, no research has examined individual change over the transition from primary to secondary school, the transition between school years in Key Stage 3 or within the PE context. Thus, it is possible that these factors may make change in achievement goal adoption and the configuration of goals more likely than that shown in previous research (Fryer \& Elliot, 2007). 


\section{Method}

\subsection{Participants and Procedures}

Data from two existing samples were used to examine individual-level and ipsative change across the transition from primary to secondary school and across the transition between year groups in Key Stage 3 of secondary school. The primary sample was comprised of 140 male $(\mathrm{N}=68)$ and female $(\mathrm{N}=72)$ participants; 11 participants were removed from the data set due to not having complete data across the transition from primary to secondary school. A total of 129 participants (62 males, 67 females) remained for analysis. Data were collected at four time points. At wave 1, the participants were nearing the end of Year 6 of primary school (July), and at waves 2-4 (October, January, July), participants had transferred into Year 7 of secondary school.

The Key Stage 3 sample was comprised of 511 male ( $\mathrm{N}=267)$ and female participants (N=244); 239 participants were removed from the data set due to not having complete data across all four measurement occasions. A total of 272 participants (125 males, 147 females) remained for analysis. Following Wave 1 (April), data were collected at 3 (July), 6 (October), and 9 (January) months and incorporated a transition between year groups between waves 2 and 3. Participants were in either Year 7, 8 or 9 for the first two measurement occasions and in Years 8, 9 and 10, respectively, at the second two measurement occasions.

In both samples, all procedures complied with the ethical guidelines of the British Psychological Society and were approved by the ethical advisory committee at the second author's institution. All procedures took place in a normal curriculum lesson. Participants were assured that all information collected would be anonymous and would remain confidential. An explanation of how to complete each section of the questionnaire was given. At each wave of measurement, each participant responded to an anonymous multi-section questionnaire which took approximately 15-20 minutes to complete. Additional details about the participants and the procedures used for data collection are available in the primary publications by Warburton and Spray $(2008,2009)$.

\subsection{Measures}

Each participant completed a multi-section questionnaire that collected the following information.

Personal details - This section of the questionnaire contained items relating to form group, date of birth, gender and age. The questionnaires were anonymous and because repeated measurements were to be taken, this information allowed participants to be identified at subsequent data points.

Goal adoption - Goal adoption was assessed using the Achievement Goals Questionnaire for Sport (Conroy et al., 2003). Pupils responded to 12 items on a seven-point Likert scale that ranged from not at all true of me (1) to very much like me (7). Three items assessed each type of goal. Sample items included, 'It is important for me to perform as well as I possibly can' (MAp: Primary sample $\alpha=.70^{1}$; Key Stage 3 sample $\alpha=.77$ ), 'I am often concerned that I may not perform as well as I can perform' (MAv: Primary sample $\alpha=.78$; Key Stage 3 sample $\alpha$ $=.80$ ), 'It is important to me to do well compared to others' (PAp: Primary sample $\alpha=.87$; Key Stage 3 sample $\alpha$ $=.84$ ) and 'I just want to avoid performing worse than others' (PAv: Primary sample $\alpha=.76$; Key Stage 3 sample $\alpha=.78)$.

Implicit theories of ability - Participants' conceptions of the nature of ability in sport and PE were assessed using the 'Conceptions of the Nature of Athletic Ability Questionnaire version 2' (CNAAQ-2, Biddle, Wang, Chatzisarantis \& Spray, 2003). Twelve items, assessing four subscales which reflect different representations of the nature of ability, were answered on a five-point Likert scale that ranged from strongly disagree (1) to strongly agree (5). Sample items included 'It is difficult to change how good you are at sport/PE' (stable), 'To be good at

1 The alphas presented for each subscale of the AGQ-S and CNAAQ-2 are the average alphas across each of the four waves of measurement. The alphas for each subscale at each wave of measurement are presented in the primary publications, Warburton and Spray (2008, 2009). 
sport/PE you need to be naturally gifted' (gift), 'You need to learn and to work hard to be good at sport/PE' (learning) and 'If you put enough effort into it, you will always get better at sport/PE' (improvement). The CNAAQ-2 posits a hierarchical factor structure, with stable and gift subscales underpinning a higher order entity belief (Primary sample $\alpha=.74$; Key Stage 3 sample $\alpha=.78$ ), and learning and improvement subscales underpinning a higher order incremental belief (Primary sample $\alpha=.74$; Key Stage 3 sample $\alpha=.78$ ). In the present study, we focused on the two higher-order dimensions of implicit beliefs.

\subsection{Data Analysis}

The extent and pattern of missing data across the four intervals were examined to see if any associations were evident with the substantive variables under investigation. Data were missing as a consequence of normal absences on the days of questionnaire administration. All main analyses were carried out using PASW Statistics 18. We followed the methods outlined in Roberts et al. (2001) and Fryer and Elliot (2007) for calculating individual-level change and ipsative continuity.

Preliminary analyses - A series of nested models testing plausible alternative factor structures was analysed at each time point for the AGQ-S and CNAAQ-2 questionnaires. These analyses assessed whether the a priori factor structure was evident at each time point. For the AGQ-S, these models tested plausible alternatives to the specified $2 \times 2$ factor structure and were consistent with the models examined in previous research on the AGQ-S (Conroy et al., 2003). For the CNAAQ-2, these models tested the hierarchical factor structure of stable and gift subscales underpinning a higher order entity factor and improvement and learning subscales underpinning a higher-order incremental factor (Biddle et al., 2003). Following confirmatory factor analysis (CFA), the factor structures of the questionnaires were tested for structural invariance across gender (primary sample) and gender and year group (Key Stage 3 sample).

We also assessed the structural stability, longitudinal factorial invariance (LFI), of the AGQ-S and CNAAQ-2 questionnaires using a series of nested models with progressively more constrained model parameters (see Conroy et al., 2003). Six separate models were assessed, one for each type of achievement goal and one for each implicit theory. The structural stability analyses enabled the assessment of whether the change over time in scores was due to true score change or change in random or specific error (Conroy et al., 2003).

Individual-level change - We used the reliable change index (RCI: Christensen \& Mendoza, 1986; Jacobson \& Truax, 1991) to determine whether the pupils' evidenced reliable change in their achievement goal adoption between any two measurement occasions. The RCI was computed for each of the four achievement goals and calculated by dividing the difference in two scores (measured at two different time points in this study) by the standard error of the difference score. Pupils were categorised as evidencing a reliable increase if their RCI was greater than 1.96, a significant decrease if their RCI was smaller than -1.96 and no change if their RCI was between -1.96 and 1.96, as values outside of this range are thought unlikely to occur by chance and are therefore indicative of reliable change (Roberts et al., 2001). To determine whether the individual-level change was due to chance, we examined the pattern of reliable change across the sample using chi-square tests and compared it to a normal distribution, (2.5\% evidencing an increase, $2.5 \%$ a decrease and $95 \%$ no change), which would be expected if change were random (Roberts et al., 2001).

We also assessed the proportion of pupils who evidenced change on different numbers of achievement goals (e.g. 1, 2, 3 or all 4 goals) by recoding the RCIs for each achievement goal at each time point comparison. Pupils who evidenced reliable change in either direction were coded as 1 and those who exhibited stability were coded as 0 . This recoded RCI was also used to test for gender differences (female $=0$ and male $=1$ ) by cross-tabulating the score with gender to determine if males or females were more likely to show reliable change on any of the four achievement goals. Finally, absolute values of the RCI for each achievement goal were correlated with scores on incremental and entity beliefs at the start of the study to determine if they predicted reliable change. 
Ipsative continuity - We calculated within-person correlations or Q correlations (Cronbach \& Gleser, 1953; Stephenson, 1952) to determine changes in profile consistency. Profile consistency coefficients were estimated by correlating each pupil's score for each achievement goal at a time point with their score for the same achievement goal at a different time point. Profile consistency estimates were assessed with a large positive coefficient indicating stability in the configuration of goals within the individual and a small positive or negative estimate reflecting a degree of change in the configuration of goals (Fryer \& Elliot, 2007). We also computed within-person standard deviations to determine changes in profile dispersion. Profile dispersion coefficients were estimated by calculating within-person standard deviations (WPSD) at each time point based on all four achievement goals and subtracting the WPSD from the initial time point from the latter time point for each time point comparison. If profile dispersion increased over time, a positive coefficient would be observed, but if profile dispersion decreased over time, a negative coefficient would be observed (Fryer \& Elliot, 2007).

Pearson product-moment correlations were used to assess any gender differences and the effects of incremental and entity beliefs at the start of the study on profile consistency and profile dispersion. Finally, we examined the significance of the profile consistency and profile dispersion estimates using the dual-hypothesis testing method of Conroy and Pincus (2006, see Fryer \& Elliot, 2007 for information). According to De Fruyt and colleagues (2006), it is important to compare within-person correlations against a randomly paired bootstrapped sample. We constructed a bootstrapped sample for comparison using the observed data. In the primary data set 13 cases were removed from the bootstrapped sample because of a lack of variance over any two time points while 33 cases were removed in the Key Stage 3 sample. In line with Fryer and Elliot (2007), and due to the mean of the bootstrapped and observed samples being similar (see Fryer \& Elliot, 2007 for explanation), the standard deviations of the WPSD in the bootstrapped and observed samples were compared.

The analyses presented below focus on key time point comparisons within the data sets. For the primary sample these are the transition from primary to secondary school (T1-T2) and the complete study (T1-T4). ${ }^{2}$ The time point comparison T1-T2 was chosen to provide a specific analysis of the immediate transition from primary to secondary school. The transition is one of the key periods in a child's school career that is likely to impact on their experiences, motivation and achievement in all school subjects, including PE. This transition occurs during an important developmental period for pupils and can be a difficult time, both academically and socially (Zeedyk et al., 2003). The time point comparison T1-T4 was chosen to give an overview of the whole study and to analyse whether changes evident in the specific transition comparison were evident a year apart.

For the Key Stage 3 sample the key time point comparisons are the transition between year groups (T2-T3) and the complete study (T1-T4). ${ }^{3}$ The time point comparison T2-T3 was chosen as it reflected the immediate transition between year groups for pupils. Galton, Gray, and Rudduck (1999) highlight in their review of transfer and transitions that there is a decline in pupil progress, motivation and enjoyment across transitions between year groups and particularly in the years between Key Stage tests (e.g., Year 7-8, Year 8-9 and Year 9-10). We wished to analyse individual-level change in motivation during this key period as well as across a longer period (T1-T4) which encompassed these transitions.

\section{Results}

\subsection{Preliminary Analyses}

These analyses have previously been reported in the primary publications (see Spray \& Warburton, 2011; Warburton \& Spray, 2008; 2009 for full details). To summarise, the AGQ-S and CNAAQ-2 were found to be valid and reliable measures of approach-avoidance achievement goals and implicit theories of ability. In both samples the CFA revealed that both the AGQ-S and the CNAAQ-2 demonstrated a good fit to the data at all

\footnotetext{
2 Data concerning other time point comparisons are available from the first author on request.

${ }^{3}$ Data concerning other time point comparisons are available from the first author on request.
} 
Warburton, V. E., \& Spray, C. M.

waves of measurement. The a priori factor structure was invariant across gender (primary sample) and gender and year group (Key Stage 3 sample). The scales in the questionnaires demonstrated acceptable longitudinal factorial invariance indicating that the change observed was true score change rather than due to random or specific error.

\subsection{Individual-Level Change}

The chi-squared statistics in Table 1 show that the pattern of reliable change was significantly different from what would have been expected if change were random for all time point comparisons. In both samples, there is evidence of both stability and reliable change, including over the two transition periods (T1-T2 and T2-T3).

Across the transition periods in both samples, most pupils reported stability in their achievement goal adoption. In the primary sample, more pupils reported a reliable decrease than a reliable increase in the adoption of all four achievement goals. The largest percentage of pupils reporting a decrease was for PAp goals, while the largest percentage for those reporting an increase was for MAv goals. For the Key Stage 3 sample, more pupils reported a reliable decrease than a reliable increase for MAp, MAv and PAv goals. The largest percentage of pupils reporting a decrease was for PAv goals, while the largest percentage for those reporting an increase was for PAp goals. The percentage of reliable changers for PAp goals was evenly distributed.

Across the study (T1-T4), most pupils reported stability in their achievement goal adoption. A greater proportion of pupils exhibited a reliable decrease than a reliable increase for all four goals in the Key Stage 3 sample and for MAp, PAp and PAv goals in the primary sample. The largest percentage of pupils reporting a reliable decrease was for PAp goals in the primary sample and MAp goals in the Key Stage 3 sample. The largest percentage of pupils reporting a reliable increase was for MAv goals in both samples. The proportion of pupils reporting a reliable increase in MAp goals in both samples was small.

\section{Table 1}

Individual-Level Change in Achievement Goals

\begin{tabular}{|c|c|c|c|c|c|c|c|c|}
\hline \multirow[b]{2}{*}{ Goal } & \multicolumn{4}{|c|}{$\mathrm{T} 1$ to $\mathrm{T} 2$} & \multicolumn{4}{|c|}{$\mathrm{T} 1$ to $\mathrm{T} 4$} \\
\hline & $\begin{array}{c}\% \\
\text { decrease }\end{array}$ & $\begin{array}{c}\% \\
\text { same }\end{array}$ & $\begin{array}{c}\% \\
\text { increase }\end{array}$ & $\chi^{2}$ & $\begin{array}{c}\% \\
\text { decrease }\end{array}$ & $\begin{array}{c}\% \\
\text { same }\end{array}$ & $\begin{array}{c}\% \\
\text { increase }\end{array}$ & $\chi^{2}$ \\
\hline MAp & 7.8 & 89.9 & 2.3 & $14.60 *$ & 10.1 & 85.3 & 4.7 & $33.30 *$ \\
\hline MAv & 12.4 & 76.7 & 10.9 & $91.13^{*}$ & 14.7 & 70.5 & 14.7 & $162.45^{*}$ \\
\hline PAp & 16.3 & 73.6 & 10.1 & $133.79 *$ & 26.4 & 65.1 & 8.5 & $324.55^{*}$ \\
\hline \multirow[t]{2}{*}{ PAv } & 12.4 & 84.5 & 3.1 & $52.29 *$ & 17.8 & 79.8 & 2.3 & $124.39 *$ \\
\hline & \multicolumn{4}{|c|}{$\mathrm{T} 2$ to $\mathrm{T} 3$} & \multicolumn{4}{|c|}{$\mathrm{T} 1$ to $\mathrm{T} 4$} \\
\hline MAp & 9.2 & 83.5 & 7.4 & $78.15^{*}$ & 20.2 & 75.7 & 4.0 & $354.87 *$ \\
\hline MAv & 11.0 & 81.3 & 7.7 & $114.22 *$ & 14.3 & 75.7 & 9.9 & $223.11 *$ \\
\hline PAp & 9.2 & 80.9 & 9.9 & $114.42 *$ & 11.8 & 79.0 & 9.2 & $149.39 *$ \\
\hline PAv & 11.8 & 80.1 & 8.1 & $133.68 *$ & 13.6 & 83.1 & 3.3 & $138.90 *$ \\
\hline
\end{tabular}

As shown in Table 2, the majority of pupils showed no reliable change on any of the achievement goals at each time point comparison suggesting a level of goal stability in the samples studied. However, the results also show that there were a proportion of pupils who evidenced change on at least one goal over the course of the study. Very few pupils showed reliable change on all four goals and none across the primary to secondary transition. No gender differences in reliable change emerged for any time point comparisons in either sample. Overall the individual-level analyses provide evidence of both goal stability and goal change. 
Predicting individual-level change - In the primary sample, incremental beliefs in Year 6 of primary school were positively associated with reliable change in MAp $(\mathrm{r}=.25, p<.01)$, PAp $(\mathrm{r}=.20, p<.05)$ and PAv $(\mathrm{r}=.18$, $p<.05)$ goals across the study (T1-T4). This suggests that those pupils high in incremental beliefs in Year 6 reported an increase in their MAp, PAp and PAv goal adoption over the course of the study. Entity beliefs were not significantly associated with reliable change in single achievement goals across the study (T1-T4). No significant associations across the transition from primary to secondary school (T1-T2) were found.

For the Key Stage 3 sample, incremental beliefs at Time 1 were negatively associated with reliable change in MAv goal adoption ( $\mathrm{r}=-.15, p<.05)$, while entity beliefs were negatively associated with reliable change in MAp goal adoption $(\mathrm{r}=-.19, p<.01)$ across the transition between year groups (T2-T3). Pupils high in incremental and entity beliefs at the start of the study therefore reported a decrease in their MAv and MAp goal adoption across the transition.

Across the study (T1-T4), incremental beliefs at Time 1 were negatively associated with reliable change in MAp $(r=-.18, p<.05)$ and PAv $(r=-.18, p<.05)$ goals. This suggests that those pupils high in incremental beliefs at the start of the study reported a decrease in their MAp and PAv goal adoption over the course of the study. Entity beliefs were not significantly associated with reliable change in single achievement goals across the study.

Table 2

Percentage of Individuals Exhibiting Reliable Change on Achievement Goals

\begin{tabular}{lcccc}
\hline & \multicolumn{2}{c}{ Primary to Secondary Transition } & \multicolumn{2}{c}{ Key Stage 3 Transition } \\
\hline Number of goals & T1 to T2 & T1 to T4 & T2 to T3 & T1 to T4 \\
\hline 0 & $51.9 \%$ & $41.1 \%$ & $55.9 \%$ & $46.3 \%$ \\
1 & $28.7 \%$ & $30.2 \%$ & $22.4 \%$ & $31.6 \%$ \\
2 & $11.6 \%$ & $18.6 \%$ & $15.4 \%$ & $13.6 \%$ \\
3 & $7.8 \%$ & $8.5 \%$ & $3.3 \%$ & $6.3 \%$ \\
4 & $0 \%$ & $1.6 \%$ & $2.9 \%$ & $2.2 \%$ \\
\hline Note. T1 to T2 = Comparison between Time 1 and Time 2. T1 to T4 = Comparison between Time 1 and Time 4. T2-T3 = Comparison \\
between Time 2 and Time 3.
\end{tabular}

\subsection{Ipsative Change}

Profile consistency - Table 3 indicates that there was evidence of goal stability in the two samples as when aggregated across the whole sample, the mean and median profile consistency scores for the primary sample were moderate and for the Key Stage 3 sample they were small to moderate for each time point comparison. For both samples the range of profile consistency scores was large. Evidence for both goal stability and goal change can be seen in Table 4. In both samples the majority of pupils reported that the configuration of goals was highly stable over time. However, there were notable proportions of pupils that evidenced some alteration (negative profile consistency) or an inversion of the initial ordering (large negative profile consistency) of their configuration of goals.

Table 3

Profile Consistency Descriptive Statistics

\begin{tabular}{lcccc}
\hline & \multicolumn{2}{c}{ Primary to secondary transition } & \multicolumn{2}{c}{ Key stage 3 transition } \\
\hline & T1 to T2 & T1 to T4 & T2 to T3 & T1 to T4 \\
\hline Mean & .35 & .40 & .31 & .24 \\
Median & .50 & .53 & .45 & .38 \\
Range & $-1.00-1.00$ & $-.97-1.00$ & $-1.00-1.00$ & $-1.00-.99$ \\
\hline
\end{tabular}

Note. $\mathrm{T} 1$ to $\mathrm{T} 2=$ Comparison between Time 1 and Time 2 . $\mathrm{T} 1$ to $\mathrm{T} 4=$ Comparison between Time 1 and Time 4 . T2-T3 = Comparison between Time 2 and Time 3. 
Warburton, V. E., \& Spray, C. M.

Table 4

Percentage of Individuals Exhibiting Different Profile Consistencies

\begin{tabular}{lcccc}
\hline & \multicolumn{2}{c}{ Primary to secondary transition } & \multicolumn{2}{c}{ Key stage 3 transition } \\
\hline & T1 to T2 & T1 to T4 & T2 to T3 & T1 to T4 \\
\hline Highly Consistent Profile & $43.1 \%$ & $41.4 \%$ & $41.2 \%$ & $38.7 \%$ \\
Negative Profile Consistency & $25.9 \%$ & $23.3 \%$ & $29.0 \%$ & $34.9 \%$ \\
Large Negative Profile Consistency & $10.3 \%$ & $1.7 \%$ & $9.9 \%$ & $11.1 \%$ \\
\hline
\end{tabular}

Note. $\mathrm{T} 1$ to $\mathrm{T} 2=$ Comparison between Time 1 and Time 2 . T1 to T4 = Comparison between Time 1 and Time 4 . T2-T3 = Comparison between Time 2 and Time 3.

In the Key Stage 3 sample a significant gender difference was found for the transition time point and indicated that girls were more consistent in their profile than boys $(r=-.16, p<.05)$ and therefore evidenced greater stability in their configuration of goals over the transition. There were no significant gender differences in profile consistency scores in the primary sample or over the entire study in both samples.

Testing the significance of profile consistency scores - The no-effect null hypothesis was rejected for each time point comparison in both samples (Primary: T1-T2, $t[115]=6.43, p<.001$; T1-T4, $t[115]=8.60, p<.001$ : Key Stage 3: $(\mathrm{T} 1-\mathrm{T} 4, t[237]=6.38, p<.001 ; \mathrm{T} 2-\mathrm{T} 3, t[237]=7.97, p<.001)$, suggesting that, on average, the level of profile consistency observed in the pupils over each time point comparison was significantly different from zero.

The chance-effect null hypothesis was tested using a randomly paired bootstrapped sample of the observed data . In both samples the means of a bootstrapped sample (Primary: T1-T2: M = .08; T1-T4: M = .09; Key Stage 3: T1-T4: $\mathrm{M}=.05$; T2-T3: $\mathrm{M}=.06$ ) were lower than those of the observed sample (Table 3). For each time point comparison, the chance-effect null hypothesis was rejected (Primary: T1-T2, $z=-3.18, p \leq .001$; $\mathrm{T} 1-\mathrm{T} 4, z=-3.92, p<.001 ;$ Key Stage 3: T1-T4, $z=-3.50, p<.001 ; \mathrm{T} 2-\mathrm{T} 3, z=-4.73, p<.001$ ), suggesting that, on average, the level of profile consistency observed in the pupils over each time point comparison had more similarity than would be expected by chance alone. The rejection of both hypotheses provides evidence of goal stability in both samples.

Predicting profile consistency - In the primary sample no significant associations were found across time point comparisons for any of the variables. In the Key Stage 3 sample entity beliefs at the start of the study were negatively associated with profile consistency coefficients (T1-T4, $\mathrm{r}=-.16, p<.05$; T2-T3, $\mathrm{r}=-.15, p<.05)$. This suggests that pupils high in entity beliefs evidenced more change in their overall configuration of goals at each time point comparison. No significant associations were found across either time point comparison for incremental beliefs.

Profile dispersion - Further evidence of goal stability is shown in Table 5. For both samples, when aggregated across the whole sample, mean profile dispersion scores were small for each time point comparison and the range of profile dispersion scores for each time point comparison was large. No significant gender differences in profile dispersion scores were found.

Table 5

Profile Dispersion Descriptive Statistics

\begin{tabular}{lcccc}
\hline & \multicolumn{2}{c}{ Primary to secondary transition } & T1 to T4 & \multicolumn{2}{c}{ Key stage 3 transition } \\
\hline & T1 to T2 & -.10 & T2 to T3 & T1 to T4 \\
\hline Mean & -.05 & $-2.12-2.37$ & -.02 & -.12 \\
Range & $-1.70-2.39$ & $-1.74-2.28$ & $-2.21-2.21$ \\
\hline
\end{tabular}

Note. $\mathrm{T} 1$ to $\mathrm{T} 2=$ Comparison between Time 1 and Time $2 . \mathrm{T} 1$ to $\mathrm{T} 4=$ Comparison between Time 1 and Time 4 . T2-T3 = Comparison between Time 2 and Time 3 . 
Testing the significance of profile dispersion scores - For both samples, the variability in the scatter was lower in the observed sample (Primary: T1-T2 $=.42$; T1-T4 $=.45$ : Key Stage 3: T1-T4 $=.34$; T2-T3 $=.31$ ) than the bootstrapped sample (Primary: T1-T2 $=.52 ; \mathrm{T} 1-\mathrm{T} 4=.47$ : Key Stage $3: \mathrm{T} 1-\mathrm{T} 4=.46$; T2-T3 $=.47$ ) for each time point comparison. In the Key Stage 3 sample these differences were significant for both time point comparisons (T1-T4, $z=-4.13, p<.001$; T2-T3, $z=-5.06, p<.001$ ), while for the primary sample these differences were significant for the T1-T2 comparison (T1-T2, $z=-2.32, p<.05$; T1-T4, $z=-.46, p>.05$ ). Except for the T1-T4 comparison in the primary sample, the results suggest that on average, the level of profile dispersion observed in the pupils over each time point comparison had more similarity than would be expected by chance alone and therefore provides evidence of goal stability.

Predicting profile dispersion - No significant associations were found across time point comparisons for any of the variables in either sample.

\section{Discussions}

The importance of understanding the temporal patterns of motivation in contexts such as PE has been underscored within the literature, not least due to the growing concerns over adolescent health and physical activity levels (Department of Health, 2011). PE has been identified as the one physical context that encapsulates all young people (Warburton \& Spray, 2009) and thus has the potential to influence young people's attitudes towards physical activity and their participation both within and beyond the curriculum (Biddle, 2001; Hagger, Chatzisarantis, Culverhouse, \& Biddle, 2003). Understanding how pupils' motivation changes over time and the factors associated with such changes are key to developing feasible interventions to ensure young people have positive experiences in school PE that can transcend physical activity contexts. The present study sought to develop the research literature on the dynamics of motivational processes in PE through employing different methods of analysing change to allow for comparisons with our previous work which has utilised mean-level analyses. We examined individual-level change and ipsative continuity within the PE context over the transition from primary to secondary school and between school years in Key Stage 3. We also sought to examine whether two key antecedents of achievement goal adoption, namely incremental and entity beliefs, and also gender, were associated with individual-level or ipsative change in achievement goal adoption.

\subsection{Stability and Change in Achievement Goals}

There was evidence of both within-person stability and change in pupils' achievement goal adoption in PE. Across the two methods of analysing individual change there was strong evidence for goal stability with the majority of pupils reporting no change in their achievement goal adoption and greater stability in the configuration of their goals. It appears that pupils' motivation in PE is fairly stable and robust and may require significant intervention from the teacher, peers or the environment to promote positive or negative change. One could assume that the transition from primary to secondary school might be considered a significant environmental intervention and thus result in greater changes in young people's motivation but that did not appear to be the case in the present research. However, future studies should consider examining the primary to secondary school transition with a larger sample size to corroborate this initial finding. This is particularly important since there appears to be some differences in the mean-level findings of studies on the primary to secondary school transition which vary in sample size (Spray et al., 2013; Warburton \& Spray, 2008).

Moreover, this evidence of goal stability differs to the findings from the educational context where the percentage of individuals exhibiting reliable change in achievement goal adoption was significantly higher than those observed in the present study, as was the percentage of individuals experiencing reliable change on three goals (Fryer \& Elliot, 2007). Our hypotheses were based on this previous research and even with the greater level of goal stability in the present study we found partial support for our hypotheses. Across both samples a greater proportion of pupils reported a decrease than an increase in MAp goal adoption while in the primary sample greater stability was evidenced in MAv goal adoption. 
The greater levels of goal stability, as evidenced by the percentage of individuals showing no reliable change in the present study, may be a result of contextual and/or sample factors. For example in the three studies cited in Fryer and Elliot (2007) the participants were undergraduate psychology students (approx. 20 years old) whose achievement goals were measured in relation to three non-cumulative exams which were five weeks apart and used a normative grading structure for their college course. The achievement goals in the present study focused on the contextual level, motivation towards PE, rather than a specific activity and with no overt, imminent assessment. Greater within-person change may be observed at the situational level, on specific activities, and also if there is an impending assessment of performance. Future research should consider these contextual issues when exploring the dynamics of motivational processes in achievement contexts.

Although the majority of pupils reported stability in their achievement goal adoption, there were a proportion of pupils in both samples who did evidence positive and negative change in their achievement goal adoption. The malleability observed in all four goals is consistent with the work of Fryer and Elliot (2007) which suggests that all four achievement goals are susceptible to change. Where we did see evidence of change, the percentages were generally larger for pupils decreasing their adoption of a particular goal. This was true for all goals and depending on which goal (e.g., MAp or PAv) is being considered will determine whether this is a positive or negative change in the pupils' motivation.

While decreases or stability in maladaptive goal strivings (MAv and PAv) could be viewed as positive changes in pupils' motivation, this is tempered by relatively few pupils reporting an increase in MAp goal striving. Across both samples, the proportion of pupils reporting an increase in MAp goals, the most adaptive type of goal striving, was small. This could be due to the nature of the PE context with the salience of competence, its public evaluation whereby it is easy to observe and evaluate pupils' ability at any moment in time, and participation in activities that are inherently competitive. This may discourage individuals from focusing on self-referenced competence. It therefore appears that adaptive changes in motivation may not occur without significant intervention and further research is needed to identify what individual or situational factors might need to be targeted to promote positive changes in motivation at the individual level. The weight of evidence in the achievement goal literature suggests we need to encourage young people to focus on self-referenced competence and identifying the characteristics of those individuals who do exhibit positive changes in their adaptive goal striving should be a priority for future research. It would be rather worrisome for those involved in educational and physical contexts if positive changes in an individual's motivation remain as elusive as they appear to be in the two samples presented.

The analysis of specific transition periods appeared to yield interesting changes in pupils' achievement goal adoption. When pupils experienced the transition between year groups in Key Stage 3, they were almost equally likely to report an increase as a decrease in their achievement goal adoption for all goals. For some pupils the transition promoted positive changes, while for others it promoted negative changes in their motivation. Exploring these individuals and their characteristics and why their motivation over the transition was either more or less positive than other pupils is important for developing our understanding of changes in motivation during school transitions.

For the primary transition, we saw both adaptive and maladaptive changes in pupils' motivation. From an adaptive perspective, the largest decrease was reported for PAp goals at both time point comparisons. This suggests that a proportion of pupils were less concerned with demonstrating normative competence. The change of peer group, teaching and PE environment that accompanies the transition to secondary school appears to discourage individuals from making comparisons to others in terms of both approach and avoidance tendencies. However, very few pupils reported increasing their focus on developing self-referenced competence. It appears that for most pupils this transition, with the change of school environment, teachers, peer group and different activities and expectations does not promote the most adaptive type of goal striving. This is worrisome given the association of this type of goal striving with a range of positive outcomes, such as higher intrinsic motivation, persistence, effort, more adaptive attributions, more effective self-regulation, greater use of effective cognitive 
Individual-level change in achievement goals in physical education

learning strategies, and adaptive help-seeking (Harwood et al., 2008; Moller \& Elliot, 2006).

\subsection{Predictors of Change in Achievement Goals}

The present study sought to identify the association of incremental and entity beliefs, and also gender, with individual-level or ipsative change in achievement goal adoption. There was no consistent pattern of gender differences across the two samples. This is in line with the work of Fryer and Elliot (2007) and the extant achievement goal literature in which the influence of gender on achievement goal adoption appears equivocal (see Meece, Glienke, \& Burg, 2006).

Within the literature, implicit theories of ability have been identified as antecedents of achievement goal adoption (e.g., Dweck, 1999; Elliot, 1999) and to influence mean-level changes in achievement goal adoption (Warburton \& Spray, 2008, 2009). Although no consistent pattern of results emerged across the two samples for the association of implicit theories with either method of analysing individual-level change, when this data is combined with our previous mean-level analyses (Warburton \& Spray, 2008, 2009) it does appear that implicit theories of ability may be important for change in achievement goal adoption.

Across both samples the analyses provided further support for the beneficial effects of encouraging pupils in PE to believe that ability can be improved and developed over time and for minimising the belief that ability is a fixed, stable quantity. Incremental and entity beliefs were associated with changes in both adaptive and maladaptive goal strivings, for example, over the immediate transition between year groups in Key Stage 3, believing that ability is a fixed, stable quantity resulted in lower levels of adaptive goal striving. This provides further evidence for the well-documented maladaptive effects of entity beliefs on goal striving (Elliot \& Church, 1997; Elliot \& McGregor, 2001; Warburton \& Spray, 2008, 2009).

However, there were a number of associations between incremental and entity beliefs that were counter to theoretical propositions and literature e.g., incremental beliefs encouraging the adoption of PAv goals. These associations highlight the risk that when PE teachers seek to foster incremental beliefs in their pupils they may inadvertently increase detrimental achievement strivings. In some pupils believing that their ability could change promoted a concern with avoiding being worse than others. This may be due to realising that if my ability can change so can others' ability. For pupils who may be low in confidence or competence in PE this could lead to maladaptive goal strivings. Future research should seek to clarify the relationships between individual-level changes in achievement goal adoption and implicit theories of ability. Incorporating other antecedents, such as perceptions of the motivational climate, perceived competence or self-esteem, will develop our understanding of the antecedents, and their interrelationships, on change in achievement goal adoption.

Interestingly, in the Key Stage 3 sample we did find support for the hypothesis that entity beliefs would be associated with goal change, as pupils who more strongly believed their ability to be a fixed, stable quantity experienced less stability in their overall configuration of goals at both time point comparisons. This is consistent with the theorising of Fryer and Elliot (2007) who suggest that because entity beliefs increase an individual's reactivity to competence feedback they are more likely to experience change in their achievement goal adoption than those who endorse incremental beliefs. However, we do not know what the changes were or whether these were positive or negative changes in the configuration. The extant literature suggests that individuals high in entity beliefs are more likely to adopt PAp and PAv goal strivings (Dweck, 1999; Elliot, 1999, 2005) and experience mostly negative consequences (Elliot \& Church, 1997; Ommundsen, 2004, 2006; Ryan \& Pintrich, 1997). Given this evidence, we might assume that the changes in goal configuration were negative with greater intensity being placed on normative-focused goals. Future research should seek to extend and clarify the current findings to determine the extent to which implicit theories of ability are associated with stability and change in achievement goal adoption. Furthermore, exploring other antecedents and consequences in relation to ipsative continuity to determine if the overall motivational profile of the individual becomes more negative over time will help elucidate which factors may be suitable candidates for inclusion in interventions to influence pupils' 
motivation in PE.

In summary, our previous work which has utilised mean-level analyses has generally indicated a negative motivational picture, particularly for adaptive goal strivings (Spray et al., 2013; Warburton \& Spray, 2008, 2009). However, the data presented indicates that there can be a substantial amount of stability in achievement goal adoption, including adaptive goal strivings, which is masked by mean-level analyses. In order to provide a more complete understanding of the dynamics of motivational processes it is important for future research to utilise different and complementary methods of analysing change.

From the data presented, it appears that there are some pupils in PE who show greater malleability in their achievement goal adoption, however, we know little about the characteristics of these individuals or the antecedents or consequences for these individuals. Future research should explore these questions using a variety of methods for assessing change and also utilise person-centred analyses to identify the characteristics of individuals who experience reliable change in their achievement goal adoption and whether there are differences between those experiencing adaptive and maladaptive changes in their motivation. We identified that incremental and entity beliefs may play a role in stability and change in achievement goal adoption, but given the extensive achievement goal literature, other factors should be examined, for example, the environmental features that help or hinder positive changes in an individual's achievement goal adoption. Moreover, we should explore whether change in one achievement goal is associated with change in other achievement goals. This line of inquiry would help in the identification of pupils who may be susceptible to positive or negative change in their motivation and in the development of future interventions to encourage positive changes in pupils' motivation in PE.

\section{References}

Anderman, L. H., \& Anderman, E. M. (1999). Social predictors of changes in students' achievement goal orientations. Contemporary Educational Psychology, 24(1), 21-37. http://dx.doi.org/10.1006/ceps.1998.0978

Barkoukis, V., Ntoumanis, N., \& Thogersen-Ntoumani, C. (2010). Developmental changes in achievement motivation and affect in physical education: Growth trajectories and demographic differences. Psychology of Sport and Exercise, 11(2), 83-90. http://dx.doi.org/10.1016/j.psychsport.2009.04.008

Barron, K. E., \& Harackiewicz, J. M. (2001). Achievement goals and optimal motivation: Testing multiple goal models. Journal of Personality and Social Psychology, 80, 706-722. http://dx.doi.org/10.1037/0022-3514.80.5.706

Biddle, S. J. H. (2001). Enhancing motivation in physical education. In G. C. Roberts (Ed.), Advances in motivation in sport and exercise (pp. 101-127). Champaign, IL: Human Kinetics.

Biddle, S. J. H., Wang, C. K. J., Chatzisarantis, N. L. D., \& Spray, C. M. (2003). Motivation for physical activity in young people: entity and incremental beliefs about athletic ability. Journal of Sports Sciences, 21, 973-989. http://dx.doi.org/10.1080/02640410310001641377

Bong, M. (2005). Within-grade changes in Korean girls' motivation and perceptions of the learning environment across domains and achievement levels. Journal of Educational Psychology, 97, 656-672. http://dx.doi.org/10.1037/0022-0663.97.4.656

Christensen, L., \& Mendoza, J. L. (1986). A method of assessing change in a single subject - An alteration of the RC index. Behavior Therapy, 17, 305-308. http://dx.doi.org/10.1016/S0005-7894(86)80060-0

Conroy, D. E., \& Pincus, A. L. (2006). A comparison of mean partialing and dual-hypothesis testing to evaluate stereotype effects when assessing profile similarity. Journal of Personality Assessment, 86, 142-149. http://dx.doi.org/10.1207/s15327752jpa8602_03

Conroy, D. E., Elliot, A. J., \& Hofer, S. M. (2003). A 2 X 2 achievement goals questionnaire for sport: Evidence for factorial invariance, temporal stability, and external validity. Journal of Sport \& Exercise Psychology, 25, 456-476.

Corker, K. S., Donnellan, M. B., \& Bowles, R. P. (2013). The development of achievement goals throughout college: Modeling stability and change. Personality and Social Psychology Bulletin, 39, 1404-1417. 
Individual-level change in achievement goals in physical education

http://dx.doi.org/10.1177/0146167213494243

Cronbach, L. J., \& Gleser, G. C. (1953). Assessing similarity between profiles. Psychological Bulletin, 50, 456-473. http://dx.doi.org/10.1037/h0057173

De Fruyt, F., Bartels, M., Van Leeuwen, K. G., De Clercq, B., Decuyper, M., \& Mervielde, I. (2006). Five types of personality continuity in childhood and adolescence. Journal of Personality and Social Psychology, 91, 538-552. http://dx.doi.org/10.1037/0022-3514.91.3.538

Department of Health. (2011). Start active, stay active: A report on physical activity from the four home countries' Chief Medical Officers.

Dweck, C. S. (1999). Self theories: Their role in motivation, personality, and development. Philadelphia, PA: Psychology Press.

Elliot, A. J. (1999). Approach and avoidance motivation and achievement goals. Educational Psychologist, 34, 169-189. http://dx.doi.org/10.1207/s15326985ep3403_3

Elliot, A. J. (2005). A conceptual history of the achievement goal construct. In A. J. Elliot \& C. S. Dweck (Eds.), Handbook of competence and motivation (pp. 52-72). New York, NY: Guildford Press.

Elliot, A. J., \& Church, M. A. (1997). A hierarchical model of approach and avoidance achievement motivation. Journal of Personality and Social Psychology, 72, 218-232. http://dx.doi.org/10.1037/0022-3514.72.1.218

Elliot, A. J., \& McGregor, H. A. (2001). A 2 X 2 achievement goal framework. Journal of Personality and Social Psychology, 80(3), 501-519. http://dx.doi.org/10.1037/0022-3514.80.3.501

Fryer, J. W., \& Elliot, A. J. (2007). Stability and change in achievement goals. Journal of Educational Psychology, 99, 700-714. http://dx.doi.org/10.1037/0022-0663.99.4.700

Galton, M., Gray, J., \& Rudduck, J. (1999). The impact of school transitions and transfers on pupil progress and attainment. London: DfES.

Hagger, M. S., Chatzisarantis, N. L. D., Culverhouse, T., \& Biddle, S. J. H. (2003). The processes by which perceived autonomy support in physical education promotes leisure-time physical activity intentions and behavior: A trans-contextual model. Journal of Educational Psychology, 95, 784-795. http://dx.doi.org/10.1037/0022-0663.95.4.784

Harwood, C. G., Spray, C. M., \& Keegan, R. J. (2008). Achievement goal theories in sport. In T. S. Horn (Ed.), Advances in sport psychology (3rd ed., pp. 157-186). Champaign, IL: Human Kinetics.

Jacobson, N. S., \& Truax, P. (1991). Clinical significance - A statistical approach to defining meaningful change in psychotherapy research Journal of Consulting and Clinical Psychology, 59, 12-19. http://dx.doi.org/10.1037/0022-006X.59.1.12

Meece, J. L., Glienke, B. B., \& Burg, S. (2006). Gender and motivation. Journal of School Psychology, 44, 351-373. http://dx.doi.org/10.1016/j.jsp.2006.04.004

Moller, A. C., \& Elliot, A. J. (2006). The 2 x 2 Achievement Goal Framework: An overview of empirical research. In A. V. Mitel (Ed.), Focus on educational psychology (pp. 307-326). Nova Science Publishers.

Muis, K. R., \& Edwards, O. (2009). Examining the stability of achievement goal orientation. Contemporary Educational Psychology, 34, 265-277. http://dx.doi.org/10.1016/j.cedpsych.2009.06.003

Ommundsen, Y. (2004). Self-handicapping related to task and performance-approach and avoidance goals in physical education. Journal of Applied Sport Psychology, 16, 183-197. http://dx.doi.org/10.1080/10413200490437660

Ommundsen, Y. (2006). Pupils' self-regulation in physical education: The role of motivational climates and differential achievement goals. European Physical Education Review, 12, 289-315. http://dx.doi.org/10.1177/1356336X06069275

Papaioannou, A., Bebetsos, E., Theodorakis, Y., Christodoulidis, T., \& Kouli, O. (2006). Causal relationships of sport and exercise involvement with goals orientations, perceived competence and intrinsic motivation in physical education: A longitudinal study. Journal of Sports Sciences, 24, 367-382. http://dx.doi.org/10.1080/02640410400022060

Roberts, B. W., Caspi, A., \& Moffitt, T. E. (2001). The kids are alright: Growth and stability in personality 
Warburton, V. E., \& Spray, C. M.

development from adolescence to adulthood. Journal of Personality and Social Psychology, 81, 670-683. http://dx.doi.org/10.1037/0022-3514.81.4.670

Roberts, G. C. (2012). Motivation in sport and exercise from an achievement goal theory perspective: After 30 years, where are we? In G. C. Roberts \& D. C. Treasure (Eds.), Advances in motivation in sport and exercise (pp. 5-58). Champaign, IL: Human Kinetics.

Ryan, A. M., \& Pintrich, P. R. (1997). "Should I ask for help?" The role of motivation and attitudes in adolescents' help seeking in math class. Journal of Educational Psychology, 89, 329-341. http://dx.doi.org/10.1037/0022-0663.89.2.329

Senko, C., \& Harackiewicz, J. M. (2005). Regulation of achievement goals: The role of competence feedback. Journal of Educational Psychology, 97, 320-336. http://dx.doi.org/10.1037/0022-0663.97.3.320

Spray, C. M., \& Warburton, V. E. (2011). Temporal relations among multidimensional perceptions of competence and trichotomous achievement goals in physical education. Psychology of Sport and Exercise, 12, 515-524. http://dx.doi.org/10.1016/j.psychsport.2011.04.007

Spray, C. M., Warburton, V. E., \& Stebbings, J. (2013). Change in physical self-perceptions across the transition to secondary school: Relationships with perceived teacher-emphasised achievement goals in physical education. Psychology of Sport and Exercise, 14, 662-669. http://dx.doi.org/10.1016/j.psychsport.2013.05.001

Stephenson, W. (1952). Some observations on Q-technique. Psychological Bulletin, 49, 483-498. http://dx.doi.org/10.1037/h0057171

Urdan, T., \& Midgley, C. (2003). Changes in the perceived classroom goal structure and pattern of adaptive learning during early adolescence. Contemporary Educational Psychology, 28, 524-551. http://dx.doi.org/10.1016/S0361-476X(02)00060-7

Warburton, V., E., \& Spray, C. M. (2008). Motivation in physical education across the primary-secondary school transition. European Physical Education Review, 14, 157-178. http://dx.doi.org/10.1177/1356336X08090704

Warburton, V., E., \& Spray, C. M. (2009). Antecedents of approach-avoidance achievement goal adoption in physical education: A longitudinal perspective. Journal of Teaching in Physical Education, 28, 214-232.

Zeedyk, M. S., Gallacher, J., Henderson, M., Hope, G., Husband, B., \& Lindsay, K. (2003). Negotiating the transition from primary to secondary school - Perceptions of pupils, parents and teachers. School Psychology International, 24, 67-79. http://dx.doi.org/10.1177/0143034303024001010 